SHORT COMMUNICATION

\title{
Memory Functioning in Post-traumatic Stress Disorder: Objective Findings versus Subjective Complaints
}

\author{
Noelle E. Carlozzi ${ }^{1,2 *^{\dagger}}$, Celinda Reese-Melancon ${ }^{3}$ \& David G. Thomas ${ }^{3}$ \\ ${ }^{1}$ University of Michigan, Ann Arbor, MI, USA \\ ${ }^{2}$ Kessler Foundation Research Center, West Orange, NJ, USA \\ ${ }^{3}$ Oklahoma State University, Stillwater, OK, USA
}

\begin{abstract}
Although memory complaints are common in post-traumatic stress disorder (PTSD), the only published study of objective and subjective memory in PTSD by Roca and Freeman indicates that subjective complaints may not accurately reflect objective performance. The present study examined memory in 21 PTSD patients, 20 combat controls and 23 non-combat controls using two objective memory measures (Rey Auditory Verbal Learning Test; Backward Digit Span) and one subjective measure (Memory Functioning Questionnaire). Analysis of variances (ANOVAs) and analysis of co-variances generally did not reveal group differences for objective memory performance. For subjective memory complaints, ANOVAs indicated group differences; PTSD participants reported more memory problems than controls. These differences disappeared, however, when depression was included as a covariate. Also, we provide some preliminary evidence that depression may mediate the relationship between PTSD symptomatology and subjective memory complaints. Findings suggest that reductions in depressive symptomatology in PTSD may be associated with decreased subjective memory complaints. Copyright (C) 2010 John Wiley \& Sons, Ltd.
\end{abstract}

Received 5 January 2010; Accepted 8 September 2010; Revised 2 July 2010

\section{Keywords}

post-traumatic stress disorder; subjective memory; objective memory; metamemory; neuropsychological assessment

\section{*Correspondence}

Noelle E. Carlozzi, Kessler Foundation Research Center, 1199 Pleasant Valley Way, West Orange, NJ 07052, USA.

†Email: ncarlozzi@kesslerfoundation.org

Published online 26 October 2010 in Wiley Online Library (wileyonlinelibrary.com) DOI: 10.1002/smi.1355

Difficulties with memory and cognition are among the leading complaints in post-traumatic stress disorder (PTSD; e.g. McNally, 1997). Objective memory problems are thought to be characteristic of PTSD (for review, see Brewin, Kleiner, Vasterling, \& Field, 2007), but the only study to examine subjective memory complaints among those with PTSD suggested that such complaints may not reflect objective memory deficits (Roca \& Freeman, 2001). Specifically, Roca and Freeman (2001) reported significant correlations between subjective memory complaints and levels of depression, symptom distress, dissociative symptoms and PTSD symptoms, but not objective memory performance. With only one study examining the relationships among these constructs in PTSD, there is a need for further research to better understand the relationship between objective and subjective memory complaints.

Due to a paucity of research examining objective and subjective memory complaints in PTSD, we provide a brief review of objective and subjective memory complaints in other populations. Specifically, the majority 
of studies examining clinical samples (e.g. mild cognitive impairment, Alzheimer's disease, temporal lobe epilepsy) do not find strong relationships between objective deficits and self-reports of cognitive dysfunction (e.g. Cook \& Marsiske, 2006; Lineweaver, Naugle, Cafaro, Bingaman, \& Lüders, 2004; Sawrie, Martin, Kuzniecky, \& Faught, 1999). In addition, the majority of studies conducted in community samples, particularly in aging community samples, have also not been able to find a strong relationship between objective and subjective memory complaints (e.g. Cook \& Marsiske, 2006; Jungwirth et al., 2004; Podewils, McLay, Rebok, \& Lyketsos, 2003; Reese \& Cherry 2006; Schmidt, Berg, \& Deelman, 2001; Zelinski, Gilewski, \& Thompson, 1980). Taken together, there is little support for strong relationships between objective and subjective memory deficits.

Although objective and subjective memory deficits are not strongly related, studies consistently find significant relationships between depression/distress and subjective memory complaints (e.g. Derouesne, Labomblez, Thibault, \& LePoncin, 1999; Jungwirth et al., 2004; Lautenschlager, Flicker, Vasikaran, Ledman, \& Almeida, 2005; Sawrie et al., 1999; Schmidt et al., 2001; Smith, Petersen, Ivnik, Malec, \& Tangalos, 1996; Zandi, 2004). Further, improvements in depression seem to be related to decreases in subjective memory complaints, but not changes in objective performance (e.g. Antikainen et al., 2001).

The present research investigated whether there is a relationship between subjective and objective memory reports among those with PTSD. Therefore, the aims of the present study were twofold. The first was to examine both objective memory performance and subjective memory beliefs to identify similarities and differences among combat veterans with PTSD and combat- and non-combat control participants. This aim extends the work of Roca and Freeman (2001) by including multiple measures of objective memory performance, as well as a measure of memory beliefs with well-established psychometric properties, and control groups for comparison purposes. The second aim was to examine the influence of depression on both objective and subjective memory complaints in a sample with PTSD. We hypothesized that participants with PTSD would indicate more objective and subjective memory problems than control participants and that controlling for the presence of depression symptomatology would decrease the relationship between objective and subjective complaints.

\section{Method}

\section{Participants}

Sixty-four males $(M$ age $=54.0$ years; $S D=5.1$; range $=44-70)$ comprised three groups: PTSD participants $(n=21)$, combat controls $(n=20)$, and noncombat controls $(n=23)$. Combat veterans with a history of PTSD were recruited from the Veterans Affairs (VA) hospital in Oklahoma City through their PTSD treatment programme. Combat veterans without PTSD and control participants were recruited through other areas of the hospital, from advertisements at VA meetings, through local veterans of foreign wars meetings, and through newspaper advertisements. Inclusion criteria were very broad; participants had to be able to read and understand English and provide informed consent. Control group participation was not restricted by substance abuse history because research suggests prevalence rates are high for co-morbid PTSD and substance abuse in veteran populations (Brown \& Wolfe, 1994). Therefore, a control group devoid of substance abuse histories would not be comparable to the typical PTSD sample. Participants ranged in age from 44 to 66 years, with an average age of 54.13 years $(\mathrm{SD}=5.1)$. There were no group differences in age.

\section{Measures}

\section{PTSD assessment}

The Clinician-Administered Post-traumatic Stress Disorder Scale-1 (CAPS; Blake et al., 1990) was administered as a structured interview to assess frequency and severity of PTSD symptoms and to provide diagnostic information on the presence/absence of PTSD. Scores on the CAPS were used to determine group status (PTSD, combat controls and normal controls) and overall PTSD symptomatology was represented by total frequency and intensity scores for PTSD (within the past month).

\section{Objective memory assessment}

The Rey Auditory Verbal Learning Test (RAVLT; Lezak, Howieson, \& Loring, 2004) was administered to examine total verbal learning (sum of words correctly recalled on trials 1-5), delayed recall (number of words correctly recalled after a 30-min delay), and recognition memory (number of words correctly recognized from a list of 50). In addition, the Backward Digit Span 
(Wechsler Memory Scales-III; Wechsler, 1997) was administered as a measure of working memory.

\section{IQ assessment}

The Kaufman Brief Intelligence Test (Kaufman \& Kaufman, 1990) measured verbal and nonverbal abilities. Participants IQ scores ranged from 70 to 117 with an average of $95.3(\mathrm{SD}=10.8)$; groups did not differ on IQ.

\section{Subjective memory assessment}

The Memory Functioning Questionnaire (MFQ; Gilewski, Zelinski, \& Schaie, 1990) assessed four aspects of metamemory: Frequency of forgetting, seriousness of forgetting, retrospective functioning and mnemonics usage. Higher scores reflect fewer forgetting incidents, less serious incidents, improvement in current memory ability relative to earlier in life and less use of mnemonics. Individual subscale scores were calculated by summing the totals of the items for each scale, with possible totals of: 231 for frequency of forgetting; 126 for seriousness of forgetting; 35 for retrospective functioning and 56 for mnemonics usage.

\section{Depression assessment}

The Beck Depression Inventory-II (BDI-II; Beck, Steer, \& Brown, 1996) is a 21-item self-report questionnaire that assesses depressive symptomatology. The BDI-II yielded one continuous score of overall depression severity, created by the summation of responses of all items; total scores ranged from 0 to 63. Participants self-reported depression scores ranged from 0 to 53 , with an average score of $20.3(\mathrm{SD}=12.7)$. Individuals with PTSD indicated higher overall self-reported levels of depression $($ mean $=29.3 ; \mathrm{SD}=12.8$ ) than either combat controls (mean $=18.15 ; \mathrm{SD}=9.2$ ) or noncombat controls $($ mean $=14.0 ; \mathrm{SD}=11.0), F(2,64)=$ $11.03, p<0.0001$.

\section{Substance abuse assessment}

The Michigan Alcohol Screening Task (MAST; Selzer, 1971) is a 25-item self-report questionnaire designed to screen for lifetime alcohol-related problems and alcoholism. Sum scores of the MAST are indicative of three categories: no alcohol problems (sum scores of $0-3$ ); a suggestion of the presence of alcoholism (scores of 4) and alcoholism indicated (scores $\geq 5$ ). Participant lifetime alcohol-related MAST scores ranged from 0 to
94 with an average score of 29.3 ( $\mathrm{SD}=19.9$ ); groups did not differ on self-reported lifetime alcohol abuse.

\section{Procedure}

All data were obtained in individual sessions conducted by a $\mathrm{PhD}$ candidate in clinical psychology, in accordance with local Institutional Review Board policies. After informed consent was obtained, the tasks and questionnaires were administered in one of four partially-counterbalanced orders. Participation took 2-3 h depending on individual response latencies. Participants were paid an honorarium of $\$ 20$.

\section{Results}

\section{Objective Memory Analyses}

An analysis of variance (ANOVA) did not indicate differences among the groups for any of our objective memory measures: $F(2,61)=2.08, p=0.13$, for RAVLT total verbal learning; $F(2,61)=0.95, p=0.39$, for RAVLT delayed recall; $F(2,61)=0.11, p=0.90$, for RAVLT recognition memory and $F(2,61)=1.33, p=$ 0.27 , for backward digit span raw score. When depression was included as a covariate, group differences were still not detected: $F(3,60)=0.18, p=0.83$, for RAVLT total verbal learning; $F(3,60)=0.79, p=0.46$, for RAVLT delayed recall; $F(3,60)=0.03, p=0.97$, for RAVLT recognition memory and $F(3,60)=0.04, p=$ 0.96 , for backward digit span raw score (see Table I for adjusted means).

\section{Subjective Memory Analyses}

The subjective memory measures were also examined. These analyses revealed significant differences among the groups on all four MFQ subscales: $F(2,61)=4.78$, $p=0.01$, for frequency of forgetting; $F(2,61)=6.27$, $p=0.003$, for seriousness of forgetting; $F(2,61)=$ $4.54, p=0.01$, for retrospective functioning and $F(2,61)=4.13, p=0.02$, for mnemonics usage (see Table II for adjusted means). Tukey comparisons indicated that participants with PTSD reported greater frequency of forgetting and seriousness of forgetting than either control group. Additionally, participants with PTSD reported more change in memory ability compared with years passed (retrospective functioning subscale) and less mnemonics usage than non-combat controls. When analyses included depression (BDI-II scores) as a covariate, all significant group differences 
Table I. Adjusted means and standard deviations for objective memory performance and subjective memory beliefs for non-combat control, combat control and PTSD participants

\begin{tabular}{|c|c|c|c|c|c|c|c|c|c|}
\hline \multirow[t]{3}{*}{ Memory } & \multicolumn{3}{|c|}{ Non-combat controls } & \multicolumn{3}{|c|}{ Combat controls } & \multicolumn{3}{|c|}{ PTSD } \\
\hline & & Covary & & & Covary & & & Covary & \\
\hline & No covariate & BDI-II & SD & No covariate & BDI-II & SD & No covariate & BDI-II & SD \\
\hline \multicolumn{10}{|l|}{ Objective } \\
\hline RAVLT total verbal learning & 40.6 & 39.0 & $(10.6)$ & 39.5 & 38.9 & $(7.1)$ & 35.1 & 37.3 & (9.5) \\
\hline RAVLT delayed recall & 6.4 & 6.4 & (3.6) & 5.5 & 5.5 & $(2.7)$ & 5.1 & 5.1 & (3.0) \\
\hline RAVLT recognition memory & 41.7 & 41.2 & $(6.5)$ & 41.4 & 41.2 & (4.6) & 40.9 & 41.6 & (4.6) \\
\hline Backward digit span & 6.4 & 6.1 & $(2.1)$ & 6.2 & 6.0 & $(2.0)$ & 5.5 & 6.0 & (1.5) \\
\hline \multicolumn{10}{|l|}{ Subjective } \\
\hline Frequency of forgetting & 144.9 & 135.4 & $(38.3)$ & 143.1 & 139.8 & (24.9) & 118.9 & 132.5 & $(25.6)$ \\
\hline Seriousness of forgetting & 71.8 & 66.6 & $(24.8)$ & 75.3 & 73.5 & (14.9) & 54.3 & 61.7 & (19.9) \\
\hline Retrospective Functioning & 17.6 & 16.8 & (6.9) & 14.9 & 14.6 & $(5.0)$ & 12.3 & 13.4 & (5.3) \\
\hline Mnemonics usage & 34.5 & 34.3 & $(10.4)$ & 34.1 & 34.0 & $(8.4)$ & 26.1 & 26.4 & (12.6) \\
\hline
\end{tabular}

Note: Bolding indicates analyses with significant group differences.

BDI-II: The Beck Depression Inventory-II; PTSD: post-traumatic stress disorder; RAVLT: Rey Auditory Verbal Learning Test.

Table II. Partial and bivariate correlations between PTSD symptomatology (reported within the last month) and measures of subjective and objective memory

\begin{tabular}{lcc}
\hline Memory & $\begin{array}{c}\text { Frequency and intensity of PTSD symptoms } \\
\text { (over the past month) }\end{array}$ \\
\cline { 2 - 3 } & $\begin{array}{c}\text { Partial correlations } \\
\text { (No covariate) }\end{array}$ & $\begin{array}{c}\text { Bivariate correlations } \\
\text { (Covary BDI-II) }\end{array}$ \\
\hline Objective & & 0.45 \\
RAVLT total verbal learning & -0.04 & 0.10 \\
RAVLT delayed recall & -0.12 & 0.12 \\
RAVLT recognition memory & -0.03 & -0.17 \\
Backward digit span & -0.23 & 0.07 \\
Subjective & & 0.13 \\
Frequency of forgetting & $-0.43^{* * *}$ & 0.11 \\
Seriousness of forgetting & $-0.38^{* *}$ & -0.05 \\
Retrospective Functioning & $-0.41^{* *}$ & \\
Mnemonics usage & $-0.35^{* *}$ & \\
\hline
\end{tabular}

${ }^{* *} p<0.01 ;{ }^{* * *} p<0.001$.

BDI-II: The Beck Depression Inventory-II; PTSD: post-traumatic stress disorder; RAVLT: Rey Auditory Verbal Learning Test.

disappeared: $F(3,60)=0.37, p=0.69$, for frequency of forgetting; $F(3,60)=1.88, p=0.16$, for seriousness of forgetting; $F(3,60)=1.57, p=0.22$, for retrospective functioning and $F(3,60)=2.73, p=0.07$, for mnemonics usage (see Table II for adjusted means).

\section{Relationship Between PTSD Symptomatology and Memory Measures}

Partial correlations were significant between the total frequency and intensity PTSD score (reported within the last month) and all measures of subjective memory, but not with any objective measures of memory. When analyses were rerun using bivariate correlations controlling for depression, all significant relationships disappeared (see Table II).

\section{Exploratory Analyses Examining Depression as a Mediator Between PTSD and Memory}

We examined the extent to which depression mediated the relationship between PTSD and subjective memory using criteria proposed by Aneschensel (2002). Find- 
Table III. Exploratory analyses examining the extent to which depression mediates the relationship between PTSD and memory $(N=64)$

\begin{tabular}{lcccccccc}
\hline & \multicolumn{2}{c}{ Frequency of forgetting } & \multicolumn{2}{c}{ Seriousness of forgetting } & \multicolumn{2}{c}{ Retrospective functioning } & \multicolumn{2}{c}{ Mnemonics usage } \\
\cline { 2 - 9 } & Model I & Model II & Model I & Model II & Model I & Model II & Model I & Model II \\
& B $(S E)$ & B $(S E)$ & B $(S E)$ & B $(S E)$ & B $(S E)$ & B $(S E)$ & B $(S E)$ & B $(S E)$ \\
\hline PTSD & $-0.43(0.11)^{\dagger}$ & $-0.05(0.13)$ & $-0.38(0.08)^{* *}$ & $-0.06(0.09)$ & $-0.41(0.02)^{* *}$ & $-0.29(0.03)$ & $-0.35(0.04)^{* *}$ & $-0.37(0.05)^{*}$ \\
BDI-II & - & $-\mathbf{5 9}(\mathbf{0 . 3 3})^{\dagger}$ & - & $-\mathbf{0 . 5 0}(\mathbf{0 . 2 4})^{* *}$ & - & $-0.18(0.07)$ & - & $0.04(0.14)$ \\
$r^{2}$ & 0.18 & 0.39 & 0.15 & 0.29 & 0.16 & 0.18 & 0.12 & 0.12 \\
$F$ & 13.96 & 19.24 & 10.61 & 12.66 & 12.19 & 6.89 & 8.53 & 4.23 \\
\hline
\end{tabular}

${ }^{*} p<0.05 ;{ }^{* *} p<0.01 ;{ }^{* * *} p<0.001 ;{ }^{\dagger} p<0.0001$.

Note: Model I: PTSD is predictor; Model II: PTSD and BDI-II predictors; BDI-II was a significant predictor of PTSD $\left(r^{2}=0.41 ; F=43.14 ; \mathrm{B}=\right.$ 0.64; $S E=0.04 ; p<0.0001$ ); Frequency of Forgetting, Seriousness of Forgetting and Restrospective Functioning were significant predictors of BDI-II; Mnemonics usages was NOT a significant predictor of BDI-II; Bolded font indicates cases where BDI-II is likely a significant mediator between PTSD and memory performance.

BDI-II: The Beck Depression Inventory-II; PTSD: post-traumatic stress disorder; RAVLT: Rey Auditory Verbal Learning Test.

ings indicated some evidence that depression mediated the relationship between PTSD and self-reported seriousness of forgetting, and PTSD and self-reported frequency of forgetting (see Table III).

\section{Discussion}

Two primary findings emerged from this research. Firstly, PTSD participants generally did not perform differently than controls on objective memory measures. Although several studies have not found group differences between controls and individuals with PTSD, many studies have demonstrated learning and memory deficits in individuals with PTSD (for review see Vasterling \& Brailey, 2005). If functional deficits do indeed exist in individuals with PTSD, it is possible that the neuropsychological tests used in this study were not sensitive enough to detect subtle differences in performance or that our sample size was not large enough to detect such differences with such large variability in scores (both within and between groups). Additional research is needed to better understand the relationship between PTSD and objective memory performance. In the extant literature, both PTSD samples and the performance measures of memory vary widely. A challenge for future research will be to get a clearer picture of how and when PTSD is associated with memory impairment.

The second major finding of the present study was that despite the general absence of group differences in objective memory performance, participants with combat-related PTSD reported more subjective memory complaints than controls. In addition, participants with PTSD reported that their memory functioning was worse than in previous years, and they reported less use of mnemonic techniques to improve memory functioning than did control participants. However, PTSD participants had significantly higher levels of depression than the control groups. When BDI-II scores were used as a covariate, these group differences in subjective memory disappeared. In addition, there was evidence that depression may mediate the relationship between subjective memory complaints (specifically seriousness of forgetting and frequency of forgetting) and PTSD symptomatology (mediation analyses in larger samples are needed to confirm this finding). These findings suggest that depressive symptoms, and not PTSD symptoms, are potentially leading to subjective memory complaints. Our results support those of Roca and Freeman (2001) who found that, among those with PTSD, subjective memory beliefs are more closely related to depression than they are to objective memory performance. Finally, consistent with Roca and Freeman, we did not find much evidence for a relationship between subjective memory complaints and objective memory scores for individuals with PTSD.

While these findings have important implications, it is also important to acknowledge some study limitations. Firstly, because our PTSD group consisted entirely of male participants with combat-related PTSD, our results may not generalize to women or other trauma samples. We also utilized four counterbalanced presentation orders in test administration which may have introduced method variance decreasing the ability to detect significant findings. Further, although the sample size utilized in this study was larger than many others in this area (e.g. Bremner et al., 1993; Gurvits et al., 1996; Uddo, Vasterling, Brailey, \& Sutker, 
1993), the high level in performance variability may have impaired our ability to detect small differences in objective memory in this size sample. Also, rates of depression were evaluated using self-report that does not allow evaluation of individuals receiving a formal diagnosis of clinical depression. Another limitation of this study is that we did not assess effort or symptom validity; studies in disability populations have shown that the frequency of insufficient effort on cognitive tasks is upwards of 20\% (Guilmette, Whelihan, Sparadeo, \& Buongiorno, 1994) and studies in treatment-seeking combat veterans indicate that more than half exhibit symptom exaggeration (e.g. Freeman, Powell, \& Kimbrell, 2008). Future studies should employ cognitive effort and symptom validity testing.

In addition, although groups did not differ in overall estimated lifetime alcohol abuse [as determined by the Michigan Alcohol Screening Task, $F(2,61)=0.01, p=$ 0.99 ], active substance abuse was likely over-represented in our control groups creating a potential confound. Participants with PTSD were recruited from treatment groups requiring abstinence prior to treatment, whereas a number of control participants were recruited from other treatment programmes not requir- ing abstinence. The well-known negative relationship between current alcohol use and cognition, as well as the improvement of cognitive deficits as early as 1 week post-sobriety, with longer periods of sobriety related to greater improvements (for review see Lezak et al., 2004) may have decreased the chances of finding objective cognitive deficits that might typically be present for those with PTSD. Ideally, future research will include groups that are more closely matched in terms of substance abuse.

In summary, self-reported memory problems appear to be more of an indicator of depression than a reliable indicator of objective memory impairment for PTSD participants. Further, there does not appear to be a strong relationship between objective and subjective memory complaints among PTSD participants. Although we have some evidence that subjective memory complaints (i.e. seriousness of forgetting and frequency of forgetting) are mediated by depressive symptomatology in PTSD, more research is needed to confirm this finding. Taken together, this research suggests that reductions in depressive symptomatology in PTSD may be associated with decreased subjective memory complaints.

\section{REFERENCES}

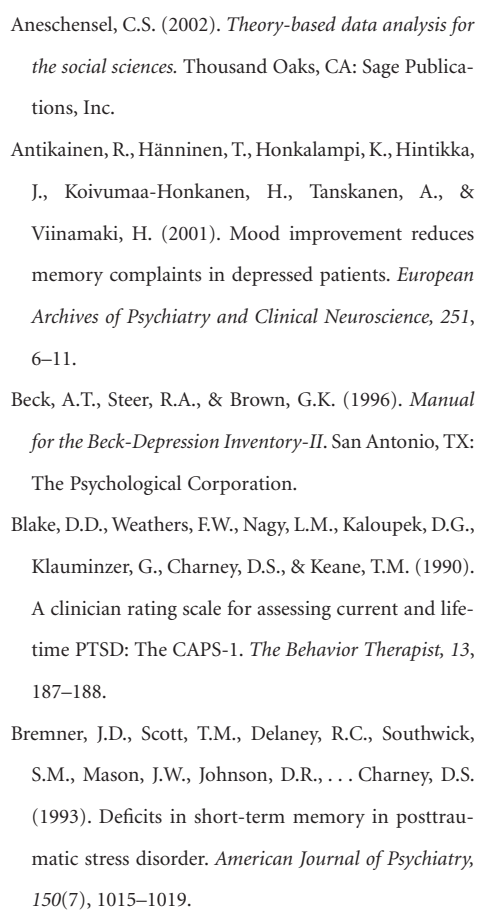

Brewin, C.R., Kleiner, J.S., Vasterling, J.J., \& Field, A.P. (2007). Memory for emotionally neutral information in posttraumatic stress disorder: A meta-analytic investigation. Journal of Abnormal Psychology, 116(3), 448-463.

Brown, P.J., \& Wolfe, J. (1994). Substance abuse, posttraumatic stress, and ethnicity. Drug \& Alcohol Dependence, 35(1), 51-59.

Cook, S., \& Marsiske, M. (2006). Subjective memory beliefs and cognitive performance in normal and mildly impaired older adults. Aging and Mental Health, 10(4), 413-423.

Derouesne, C., Labomblez, L., Thibault, S., \& LePoncin, M. (1999). Memory complaints in young and elderly subjects. International Journal of Geriatric Psychiatry, 14, 291-301.

Freeman, T., Powell, M., \& Kimbrell, T. (2008). Measuring symptom exaggeration in veterans with chronic posttraumatic stress disorder. Psychiatry Research, 158, 374-380.

Gilewski, M.J., Zelinski, E.M., \& Schaie, K.S. (1990). The Memory Functioning Questionnaire for assessment of memory complaints in adulthood and old age. Psychology and Aging, 5(4), 482-490.

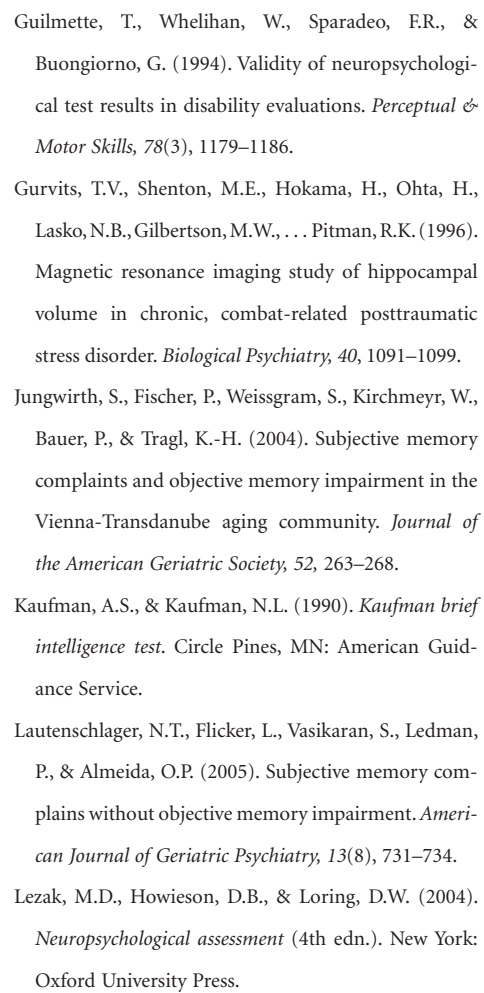


Lineweaver, T.T., Naugle, R.I., Cafaro, A.M., Bingaman, W., \& Lüders, H. (2004). Patients' perceptions of memory functioning before and after surgical intervention to treat medically refractory epilepsy. Epilepsia, 45(12), 1604-1612.

McNally, R.J. (1997). Implicit and explicit memory for trauma-related information in PTSD. Annals of the New York Academy of Sciences, 821, 219-224.

Podewils, L.J., McLay, R.N., Rebok, G.W., \& Lyketsos, C.G. (2003). Relationship of self-perceptions of memory and worry to objective measures of memory and cognition in the general population. Psychosomatics, 44(6), 461-470.

Reese, C.M., \& Cherry, K.E. (2006). Effects of age and ability on self-reported memory functioning and knowledge of memory in aging. Journal of Genetic Psychology, 167(2), 221-240.

Roca, V., \& Freeman, T.W. (2001). Complaints of impaired memory in veterans with PTSD. The American Journal of Psychiatry, 158 (10), 1738-1739.
Sawrie, S.M., Martin, R.C., Kuzniecky, R., \& Faught, E. (1999). Subjective versus objective memory change after temporal lobe epilepsy surgery. Neurology, 53(7), 1511-1517.

Schmidt, I.W., Berg, I.J., \& Deelman, B.G. (2001). Relations between subjective evaluations of memory and objective memory performance. Perceptual and Motor Skills, 93(3), 761-776.

Selzer, M.L. (1971). The Michigan Alcoholism Screening Test: The quest for a new diagnostic instrument. American Journal of Psychiatry, 127(12), 1653-1658.

Smith, G.E., Petersen, R.C., Ivnik, R.J., Malec, J.F., \& Tangalos, E.G. (1996). Subjective memory complaints, psychological distress, and longitudinal change in objective memory performance. Psychology and Aging, 1(2), 272-279.

Uddo, M., Vasterling, J.J., Brailey, K., \& Sutker, P.B. (1993). Memory and attention in combat-related post-traumatic stress disorder (PTSD). Journal of Psychopathology and Behavioral Assessment, 15(1), 43-52.
Vasterling, J.J., \& Brailey, K. (2005). Neuropsychological findings in adults with PTSD. In J.J. Vasterling, \& C.R. Brewin (Eds.), Neuropsychology of PTSD (pp. 178207). New York: The Guilford Press.

Wechsler, D. (1997). Wechsler Memory Scale (3rd edn.). San Antonio, TX: The Psychological Corporation.

Zandi, T. (2004). Relationship between subjective memory complaints, objective memory performance, and depression among older adults. American Journal of Alzheimers Disease and Other Dementias, 19(6), 353-360.

Zelinski, E.M., Gilewski, M.J., \& Thompson, L.W. (1980). Do laboratory tests relate to self-assessment of memory in the young and old? In L.W. Poon, J.L. Fozard, L.S. Cermak, D. Arenberg, \& L.W. Thompson (Eds.), New directions in memory and aging: Proceedings of the Geogre Talland Memorial Conference (pp. 519-544). Hillsdale, NJ: Lawrence Erlbaum Associates. 